\title{
Metallomics: an emerging interdisciplinary science
}

\author{
Michael Sperling • Uwe Karst
}

Published online: 22 December 2012

(C) Springer-Verlag Berlin Heidelberg 2012

For many years, the function and fate of metals and their species in biological systems has been of increasing interest to the scientific community, because of their important physiological effects and toxic properties. Although different groups of scientists, including biologists, inorganic chemists, physicians, and analytical chemists, have all attempted to answer questions related to metal species in the body by use of their established scientific methods and instrumentation, it has become clear that much more intense interdisciplinary cooperation between scientists of all these disciplines is required to successfully address the highly complex questions arising from their research. The subject "metallomics" has emerged with the objective of providing a deep understanding of the kind, concentration, and function of metal species in biology. Although "omics" is typically understood to mean a comprehensive approach to the target analytes, it is more realistic to include all methods leading toward this objective in metallomics. However, it is obvious that progress in this field is crucially dependent on progress in analytical chemistry and, in particular, in speciation analysis.

Furthermore, interdisciplinary cooperation between analytical chemists on the one side and biologists or physicians on the other must be raised to another level. The classical picture of the analytical chemist who receives samples from a colleague from the life sciences, determines the concentration of one or more previously assigned analytes, and returns concentration data to the colleague, definitely needs refinement to enable

Published in the topical collection Metallomics with guest editors Uwe Karst and Michael Sperling.

M. Sperling $\cdot$ U. Karst $(\bowtie)$

Institute of Inorganic and Analytical Chemistry,

University of Münster, Corrensstr. 30,

48149 Münster, Germany

e-mail: uk@uni-muenster.de

\section{Sperling}

European Virtual Institute for Speciation Analysis (EVISA),

Mendelstr. 11,

48149 Münster, Germany successful cooperation in the highly complex world of metallomics. Involvement of analytical chemists will be required from the earliest stages of project planning to ensure that all possibilities of the most recent (hyphenated) analytical techniques will be exploited and that analytical challenges are addressed appropriately throughout the project.

To facilitate communication between both sides, a dedicated conference series was established a few years ago and, after three conferences in Kyoto, Japan, in 2007, Cincinnati, USA, in 2009, and Münster, Germany, in 2011, a fourth will be held in Oviedo, Spain, in the summer of 2013. In addition to this, dedicated literature, e.g. special issues of broadly oriented analytical journals, is particularly helpful in promoting the subject and involving other colleagues from academia and industry.

The purpose of this special issue is to cover the broad range of topics from the life sciences related to the new field of metallomics. A two-digit number of high-quality articles discusses such questions as the metabolism of metals, the involvement of metal species in dysfunction and diseases, the development of metallodrugs and characterization of their activity, and the involvement of metal species in gene expression and regulation. An equally broad range of analytical techniques has been used to determine the interactions of metal species and biomolecules to provide the respective answers. These techniques include spectroscopic methods (Mössbauer spectroscopy, NMR, FTIR, EPR), techniques hyphenated with mass spectrometric detection (LC-ICPMS, CE-ICP-MS, LC-ESI-MS), imaging techniques ( $\mu$ XRF, SR-XRF, XANES, X-ray tomography, SIMS, LAICP-MS, fluorescence microscopy), separation techniques (HPLC, PAGE), and, last but not least, bioanalytical techniques, for example PCR and gene-expression analysis. We would like to thank all the authors who responded to our invitation and submitted their exciting contributions.

We hope this special issue will be a valuable source of information for all colleagues with an interest in this highly interdisciplinary research field. Let us wish the readers an interesting, pleasant, and rewarding experience with this special issue on metallomics. 


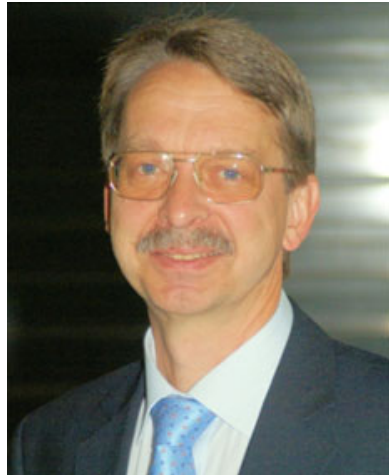

Michael Sperling is Chief Executive Officer of the European Virtual Institute of Speciation Analysis (EVISA), an association formed to promote speciation analysis, especially by fostering interdisciplinary cooperation, transfer of know-how, education, and exchange of information. He gained his broad experience in atomic spectrometry and trace element and speciation analysis as the head of the laboratory for applied research of a renowned instrument manufacturer. More recently, Michael Sperling joined the Institute of Inorganic and Analytical Chemistry of the University of Münster, where he is investigating techniques and methods for speciation analysis. His main research interest is the development of hyphenated analytical techniques for study of metal species in biological systems.

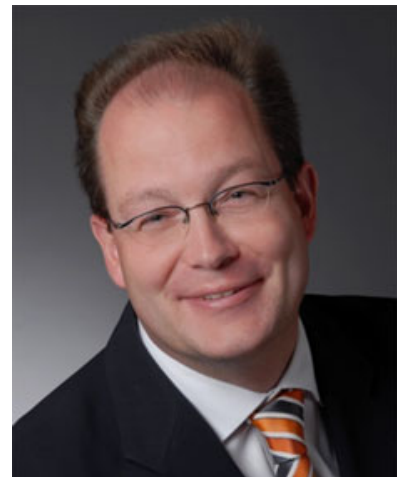

Uwe Karst received his $\mathrm{PhD}$ at the University of Münster, Germany, in Karl Cammann's group and moved to the University of Colorado in Boulder for a postdoctoral fellowship with Robert E. Sievers. After finishing his Habilitation in Münster, he was appointed Full Professor of Chemical Analysis at the University of Twente, The Netherlands. In 2005, he took his current position as Chair of Analytical Chemistry in Münster. His main research interests include hyphenated techniques, with particular focus on pharmaceutical analysis, elemental speciation, and metallomics. 